\title{
Scalable Implementations of MPI Atomicity for Concurrent Overlapping I/O
}

\author{
Wei-keng Liao \\ Alok Choudhary \\ Kenin Coloma \\ George K. Thiruvathukal \\ Loyola University Chicago, gkt@cs.luc.edu \\ Lee Ward
}

See next page for additional authors

Follow this and additional works at: https://ecommons.luc.edu/cs_facpubs

Part of the Computer and Systems Architecture Commons, Computer Sciences Commons, and the Data Storage Systems Commons

\section{Recommended Citation}

W. Liao et al., "Scalable Implementations of MPI Atomicity for Concurrent Overlapping I/O," in International Conference on Parallel Processing, 2003.

This Conference Proceeding is brought to you for free and open access by the Faculty Publications and Other Works by Department at Loyola eCommons. It has been accepted for inclusion in Computer Science: Faculty Publications and Other Works by an authorized administrator of Loyola eCommons. For more information, please contact ecommons@luc.edu.

\section{(c) () $(9)$}

This work is licensed under a Creative Commons Attribution-Noncommercial-No Derivative Works 3.0 License. Copyright $\odot 2003$ Wei-keng Liao, Alok Choudhary, Kenin Coloma, George K. Thiruvathukal, Lee Ward, Eric Russell, and Neil Pundit 


\section{Authors}

Wei-keng Liao, Alok Choudhary, Kenin Coloma, George K. Thiruvathukal, Lee Ward, Eric Russell, and Neil Pundit 


\title{
Scalable Implementations of MPI Atomicity for Concurrent Overlapping I/O
}

\author{
Wei-keng Liao ${ }^{\dagger}$, Alok Choudhary ${ }^{\dagger}$, Kenin Coloma ${ }^{\dagger}$, George K. Thiruvathukal ${ }^{\ddagger}$, \\ Lee Ward*, Eric Russell*, and Neil Pundit* \\ $\dagger$ ECE Department \\ Northwestern University \\ $\ddagger$ CS Department \\ Loyola University \\ * Scalable Computing \\ Systems Department \\ Sandia National Laboratories
}

\begin{abstract}
For concurrent I/O operations, atomicity defines the results in the overlapping file regions simultaneously read/written by requesting processes. Atomicity has been well studied at the file system level, such as POSIX standard. In this paper, we investigate the problems arising from the implementation of MPI atomicity for concurrent overlapping write access and provide a few programming solutions. Since the MPI definition of atomicity differs from the POSIX one, an implementation that simply relies on the POSIX file systems does not guarantee correct MPI semantics. To have a correct implementation of atomic I/O in MPI, we examine the efficiency of three approaches: 1) file locking,

2) graph-coloring, and 3) process-rank ordering. Performance complexity for these methods are analyzed and their experimental results are presented for file systems including NFS, SGI's XFS, and IBM's GPFS.

\section{Introduction}

Concurrent file access has been an active research topic for many years. Efforts were contributed in both software development as well as hardware design to improve the I/O bandwidth between computational units and storage systems. While most of these works only consider exclusive file access among the concurrent I/O requests, more scientific applications nowadays require data partitioning with
\end{abstract}


overlap among the requesting processes $[1,2$, 3, 4]. For instance, ghost cells are commonly used in multi-dimensional array partitioning such that the sub-array partitioned in one process overlaps with its neighbors near the boundary. A couple examples that use this ghosting technique are large scale simulations in earth climate and N-body astrophysics, hydrodynamics using Laplace equations, both where a strong spatial domain partitioning relationship is present. Figure 1 illustrates an example of a two-dimensional array in a block-block partitioning pattern, where a ghost cell represent data "owned" by more than one process. A typical run of this large-scale type of applications can take from days to months and usually outputs data periodically for the purposes of check-pointing as well as progressive visualization. During check-pointing, the output of ghost cells creates overlapping I/O from all processes concurrently. The outcome of the overlapped file regions from a concurrent $\mathrm{I} / \mathrm{O}$ is commonly referred as atomicity.

In this paper, we examine the implementation issues for concurrent overlapping I/O operations that abide the MPI atomicity semantics. We first differentiate the MPI atomicity semantics from the definition in POSIX standard. The POSIX definition only considers atomicity at the granularity of read ()/write () calls in which only a contiguous file space can be specified in a single I/O request. In MPI, a pro-

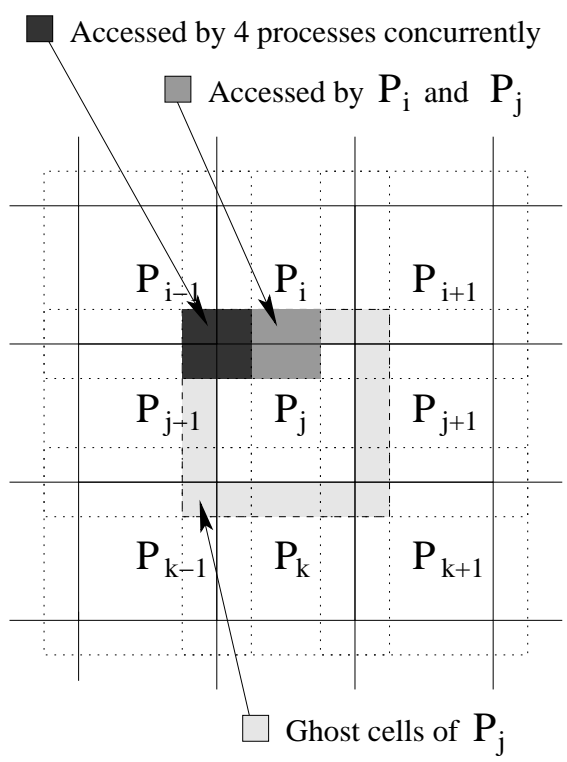

Figure 1: An example of 2D array partitioning with overlapping at the boundary. The ghost cells of $P_{j}$ overlaps with its 8 neighbor processes which results some areas are accessed by more than one processes simultaneously.

cess can define a non-contiguous file view using MPI derived data types and subsequent I/O calls can then implicitly access non-contiguous file regions. Since the POSIX definition is not aware of non-contiguous I/O access, it alone cannot guarantee atomic access in MPI, and additional efforts are needed above the file system to ensure the correct implementation of atomic MPI access. In this work, we study two approaches for atomicity implementation: using byte-range file locking and a process handshaking strategy. Using a byte-range file locking mechanism is a straightforward method to ensure the atomicity. In many situations, however, file locking can 
serialize what were intended to be concurrent $\mathrm{I} / \mathrm{O}$ calls and, therefore, it is necessary to explore alternative approaches. Process handshaking uses inter-process communication to determine the access sequence or agreement on the overlaps, in which two methods are studied: graph-coloring and process-rank ordering methods. These two methods order the concurrent $\mathrm{I} / \mathrm{O}$ requests in a sequence such that no two overlapping requests can perform at any instance. Experimental performance results are provided for running a test code using a column-wise partitioning pattern on three machine platforms: an Linux cluster running an extended NFS file system, an SGI Origin2000 running XFS, and an IBM SP running GPFS. The results show that, in general, using file locking generates the worst performance and using the process-rank ordering performs the best on all three machines.

\section{Concurrent Overlapping I/O}

The concurrent overlapping $I / O$ referred to in this paper occurs when I/O requests from multiple processes are issued simultaneously to the file system and overlaps exist among the file regions accessed by these requests. If all the requests are read requests, the file system can use the disk cache to duplicate the overlapped data for the requesting processes. In this case, there is no conflict in obtaining file data among the overlapping processes. However, when one or more I/O requests are write requests, the outcome of the overlapped regions, either in file or in process's memory, can vary depending on the implementation of the file system. This problem is commonly referred as the $I / O$ atomicity.

\subsection{POSIX Atomicity Semantics}

POSIX standard defines atomicity such that all the bytes from a single file $\mathrm{I} / \mathrm{O}$ operation that start out together end up together, without inter-

The rest of the paper is organized as follows. Section 2 describes the MPI atomicity semantics and its difference from the POSIX definition. We explore three potential approaches for implementing MPI I/O atomicity in depth in Section 3. In Section 4, we present performance results on three parallel file systems. The paper is concluded in Section 5. I/O operations confined by this definition include the system calls that operate on regular files, such as open(), read(), write(), chmod (), lseek (), close (), and so on. In this paper, we focus on the the effect of the read and write calls on the atomicity.

The POSIX definition can be simply inter- 
preted as that either all or none of the data written by a process is visible to other processes. The none case can be either the write data is cached in a system buffer and has not been flushed to the disk or the data is flushed but over-written by other processes. Hence, when POSIX semantics is applied to the concurrent overlapping I/O operations, the data resulted in the overlapped regions in disk shall consist of data from only one of the write requests. In other words, no interleaved data from more than one process shall appear in the overlapped regions. Otherwise, in non-atomic mode, the result of the overlapped region is undefined, i.e. it may comprise mixed data from more than one write request. Many existing file systems support the POSIX atomicity semantics, such as NFS, UFS, IBM PIOFS, GPFS, Intel PFS, and SGI XFS.

POSIX atomicity mainly considers the I/O calls defined within the POSIX scope in which its read and write calls share a common characteristic: one $\mathrm{I} / \mathrm{O}$ request can only access a contiguous file region specified by a file pointer and the amount of data starting from the pointer. Therefore, the overlapped data written by two or more POSIX I/O calls can only be a contiguous region in file. Many POSIX file systems implement the atomic I/O by serializing the process of the requests such that the overlapped regions can only be accessed by one process at any moment. By considering only the contiguous file access, the POSIX definition is suitable for file systems that mainly handle non-parallel I/O requests. For I/O requests from parallel applications that frequently issue non-contiguous file access requests from multiple process, POSIX atomicity may improperly describe such parallel access patterns and impose limitation for the I/O parallelism.

\subsection{MPI Atomicity Semantics}

MPI standard 2.0 [7] extends its atomicity semantics by taking into consideration of the parallel I/O operations. The MPI atomic mode is defined as: in concurrent overlapping MPI I/O operations, the results of the overlapped regions shall contain data from only one of the MPI processes that participates in the I/O operations. Otherwise, in the MPI non-atomic mode, the result of the overlapped regions is undefined. The major difference of the MPI atomicity from POSIX definition lies on the use of MPI file view, a new file concept introduced in MPI 2.0. A process' file view is created by calling MPI_File_set_view() through an MPI derived data type that specifies the visible file range to the process. When used in message passing, the MPI derived data type is a powerful mechanism for describing the memory layout of a message buffer. This convenient tool is extended in MPI 2.0 for describing the file layout for process' file view. Since a derived data 
type can specify a list of non-contiguous file regions, the visible data to a process can also be non-contiguous. When performing an MPI I/O request, the visible regions are logically considered as a continuous data stream coming/going from/to the file system to/from the requesting process.

Similar to POSIX, MPI atomicity considers a call to MPIFFile_read_xxx()/ MPI_File_writ_xxx() as a single I/O request. Unlike overlap in POSIX I/O, the overlapped file regions between two processes can also be non-contiguous in file, generating multiple overlapping file regions in one MPI I/O call. If the underlying MPI I/O implementation considers the access to each contiguous file segment as a single read ()/write () call to the file system, then there will be multiple calls issued simultaneously from a process to the file system. Although the atomicity of accessing to a contiguous overlapped region is guaranteed in the POSIX compliant file systems, the MPI atomicity which demands atomicity across one or more regions of overlap cannot simply rely on the POSIX I/O calls. Additional effort is required to implement a correct MPI atomicity semantics. The fact that MPI derived data types provide more programming flexibility when specifying non-contiguous file layout increases the complexity of enforcing atomicity in MPI on POSIX file systems.

Figure 2 shows an example of two concurrent

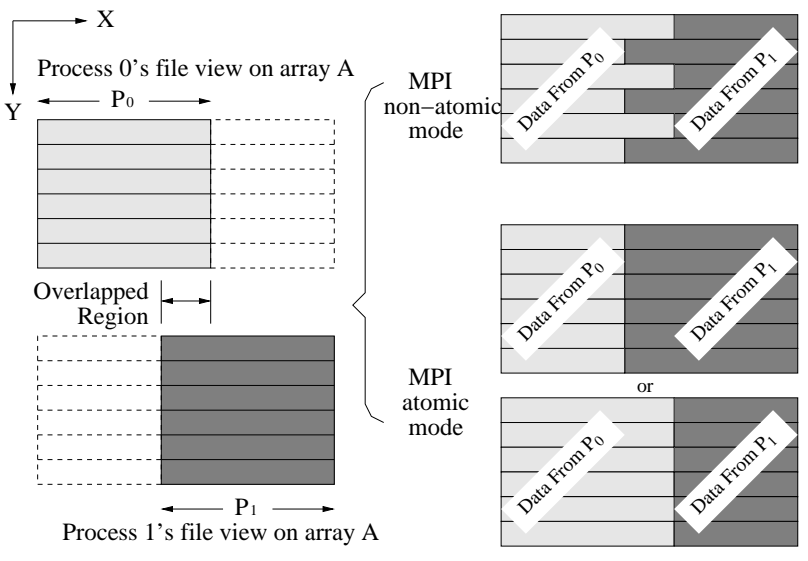

Figure 2: An example of two concurrent overlapping writes where a 2D array is partitioned column wise with a few columns overlapped. In the MPI atomic mode, the data in the overlapped region can only come from either one of the processes. In the non-atomic mode, the result is undefined, for example, interleaved.

MPI write requests in atomic and non-atomic modes. The file views of both processes, $P_{0}$ and $P_{1}$, consist of 6 non-contiguous file segments, assuming the two-dimensional array is stored in row major. If the MPI implementation considers writing each of the file segment as a single call to write(), then there will be 12 write requests in total and the processing order of these requests in the file system can be arbitrary. The result in the overlapped columns can, hence, contain interleaved data, as illustrated in the MPI non-atomic mode. Even with POSIX atomicity, the same outcome will occur in a POSIX file system since it only considers the read () /write () call individually. 
Therefore, the MPI implementation cannot simply rely on the file system to provide the correct file atomicity.

\section{Implementation Strategies}

The design of existing file systems seldom consider concurrent overlapping $\mathrm{I} / \mathrm{O}$ requests and many implementation strategies that are intended for performance enhancement actually hinder the parallelism of overlapping I/O. For example, in most client-server type of file systems, strategies such as read-ahead and writebehind are adopted in which read-ahead prefetches several file blocks following the data actual requested to the client's system cache in anticipation of program's sequential reading pattern and write-behind accumulates several requests in order to better utilize the available $\mathrm{I} / \mathrm{O}$ bandwidth. The read-ahead and write-behind policies often work against the goals of any file system relying on random-access operations which are used commonly in parallel I/O operations. Under the use of these two policies, two overlapping processes of a concurrent I/O operation can physically cache more overlapping data than logically overlaps in their file views. It is also possible that the overlapping data of two processes is cached by other processes because of the read ahead.

The file cache consistency problem has been studied extensively in many client-server based file systems. The most commonly implemented caching scheme is to consult the server's modification time for the data cached on the clients before issuing the $\mathrm{I} / \mathrm{O}$ requests to the servers. Obviously, communication overhead between server and clients for cache validation and refreshing can become significant for a concurrent overlapping I/O request due to the unnecessary data transfers. Although this problem can be alleviated by disabling the use of readahead/write-through, the performance gain of the reduced overhead may not offset the performance loss of disabling caching. In this work, our discussion is not limited to specific file systems and we assume the general I/O requests can start at arbitrary file space. We now examine two potential implementation strategies for MPI atomicity and analyze their performance complexity:

1. Using byte-range file locking - This approach uses the standard Unix byterange file locking mechanism to wrap the $\mathrm{read} / \mathrm{write}$ call in each process such that the exclusive access permission of the overlapped region can be granted to the requesting process. While a file region is locked, all read/write requests to it will directly go to the file server. Therefore, the written data of a process is visible to other processes after leaving the locking mode and the subsequent read requests will always 
obtain flesh data from the servers because of the use of the read locks.

2. Using process handshaking - This approach uses MPI communication to perform inter-process negotiation for writing to the overlapped file regions. The idea is a preferable alternative to using file locking. However, for file systems that perform read-ahead and write-behind, a file synchronization call immediately following every write call is required to flush out all information associated with the writes in progress. A cache invalidation shall also perform in each process before reading from the overlapped regions such that the flesh data is obtained from servers. Under this strategy category, we further discuss two negotiation methods: graph-coloring and process-rank ordering.

In order to help describing the above three approaches in terms of data amount and file layouts, we use two concurrent overlapping $\mathrm{I} / \mathrm{O}$ cases as examples. These two cases employ commonly seen access patterns in many scientific applications: row-wise and column-wise partitioning on a two-dimensional array.

\subsection{Row and Column-wise 2D Ar- ray Partitioning}

Given $P$ processes participating a concurrent $\mathrm{I} / \mathrm{O}$ operation, the row-wise partitioning pattern divides a two-dimensional array along its most significant axis while the column-wise divides it along the least significant axis. The following assumptions are used in these partitioning examples:

- All $P$ processes concurrently write their sub-arrays to a single shared file.

- The layouts of the 2-dimensional array in process' memory and disk storage are in row-major order where axis $Y$ is the most significant axis and $X$ is the least.

- The sub-arrays partitioned in every two processes with consecutive rank id numbers overlap with each other for a few rows/columns on the boundary along the partitioning axis.

- The global array is of size $M \times N$ and the number of overlapped rows/columns is $R$, where $R<M / P$ and $R<N / P$.

- To simplify the discussion, we assume all $\mathrm{I} / \mathrm{O}$ requests are write requests.

Figure 3 illustrates the two partitioning patterns on $P=4$ processes. In the row-wise case, the file view of process $i$ is a sub-array of size $M^{\prime} \times$ 


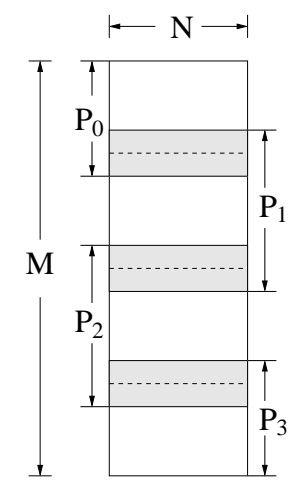

(a) Row-wise partitioning
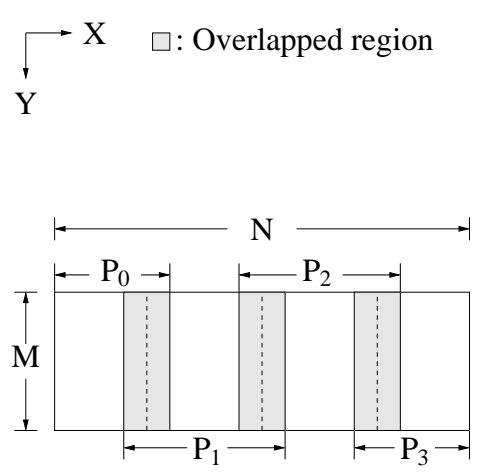

(b) column-wise partitioning
Figure 3: Row-wise and column-wise partitioning on a 2-dimensional array. The file views of every two consecutive processes overlap with each other in $R$ rows/columns along $Y / X$ axis.

$N$, where $M^{\prime}=\frac{M}{P}+R$, if $0<i<P-1$. In the column-wise case, the file view of process $i$ is of size $M \times N^{\prime}$, where $N^{\prime}=\frac{N}{P}+R$ for $0<i<P-1$. For the process $i=0$ or $P-1$, the file view contains $\frac{R}{2}$ rows/columns less in row and column-wise cases, respectively.

\subsection{Byte-range File Locking}

The byte-range file locking is a mechanism provided by a file system within its locking protocol. This mechanism can be used to ensure the exclusive access to a locked file region. If a set of concurrent I/O calls contains only read requests, the locking protocol is usually implemented to allow a shared read lock so that more than one process can read the locked data simultaneously. If at least one of the I/O requests is a write request, the write lock is often granted exclusively to the requesting processes. Most of the existing locking protocols is central managed and its scalability is, hence, limited. A distributed locking protocol used in the IBM GPFS file system improves the performance of granting locking requests by having a process manage its granted locked file region for the further requests from other processes [8]. When it comes to the overlapping requests, however, concurrent writes to overlapped data must be still sequential.

Row-wise Partitioning We now use the rowwise partitioning example shown in Figure 3(a) to describe the atomicity implementation using file locking. In this example, the file view of a process overlaps $R$ rows with its previous and successive processes. Since the file storage layout is assumed to be in a row-major order, i.e. each row of size $N$ is stored consecutively to its previous and successive row, every process' file view actually covers a contiguous file space. Therefore, the concurrent overlapping I/O can be implemented using a single write () call in each process. On a file system that supports only the atomic mode, atomic file results are automatically guaranteed for the row-wise partitioning case. On file systems that do no support the atomic mode, wrapping the I/O call in each process with byte-range locking of the file region will also generate atomic results. ROMIO, an MPI-IO implementation developed at Argonne 
National Laboratory, relies on the use of byterange file locking to implement the correct MPI atomicity in which processes must obtain a exclusive write lock to the overlapped file regions before perform the write $[9,10]$.

Column-wise Partitioning In the columnwise partitioning case shown in Figure 3(b), the file view of each process is a sub-array of size $M \times N^{\prime}$ overlapping $R$ columns with its left and right processes. Each of the $M$ rows of size $N^{\prime}$ in the file view is not contiguous with its previous or successive row in the file storage layout. The distance between the first elements of two consecutive rows in each process' file view is $N$. Therefore, the overlapped file regions of two consecutive processes consists of $M$ noncontiguous rows of size $R$ each. Figure 4 shows an MPI code fragment that creates the file view for each process using a derived data type to specify the column-wise partitioning pattern and uses a collective MPI-IO call to perform the concurrent write.

A straightforward implementation for the column-wise case is to regard each contiguous I/O request as a single read () /write () call. This approach results $M$ write calls from each process and $P M$ calls in total. On a POSIX file system, if the $P M$ requests are processed concurrently without any specific order, interleaved results may occur in the overlapped regions. Since processing order of these write requests

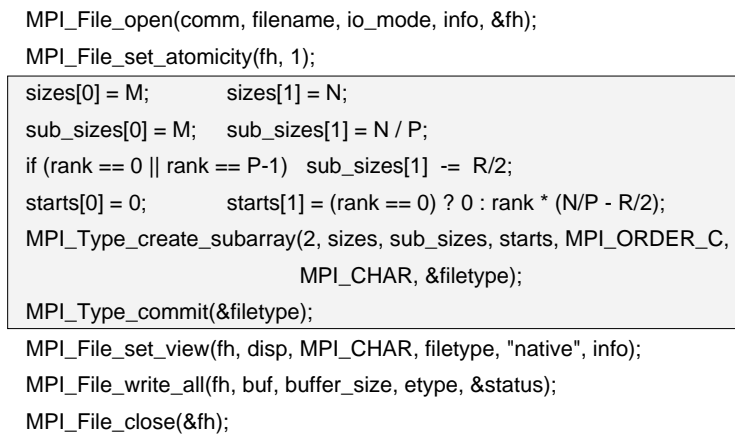

Figure 4: An MPI code fragment that performs the column-wise access. The shade area illustrates how to create the derived data type, filetype, which is used to define process's file view at line 10.

can be arbitrary, the same scenario can also occur on other file systems even if file locking wraps around each I/O call. Enforcing the atomicity of individual read ()/write () calls is not sufficient to enforce MPI atomicity. In order to do so, the file lock must start at the process's first file offset and end at the very last file offset the process will write, virtually the entire file. All $M$ rows of the overlapped region will be accessed atomically.

Though POSIX defines a function, lio_listio(), to initiate a list of noncontiguous file accesses in a single call, it does not explicitly indicate if its atomicity semantics are applicable. If POSIX atomicity is extended to lio_listio(), the MPI atomicity can be guaranteed by implementing the noncontiguous access on top of lio_listio(). Otherwise, additional effort such as file locking 
is necessary to ensure the MPI atomicity.

\subsection{Processor Handshaking}

An alternative approach to avoid using file locking is through process handshaking. Through inter-process communication the overlapping processes negotiate with each other to obtain the desirable access sequence to the overlapped regions. In this section, we discuss two possible implementations of process handshaking: graph-coloring and process-rank ordering methods.

\subsubsection{Graph-coloring Approach}

Given an undirected graph $G=(V, E)$ in which $V$ represents a set of vertices and $E$ represents a set of edges that connect the vertices, a $k$ coloring is a function $C: V \rightarrow\{1,2, \ldots k\}$ such that for all $u, v \in V$, if $C(u)=C(v)$, then $(u, v) \notin E$; that is, no adjacent vertices have the same color. The graph-coloring problem is to find the minimum number of colors, $k$, to color a given graph. Solving the MPI atomicity problem can be viewed as a graph-coloring problem if the $\mathrm{I} / \mathrm{O}$ requesting processes are regarded as the vertices and the overlapping between two processes represents the edge. When applying graph-coloring to the MPI atomicity implementation, the I/O processes are first divided into $k$ groups (colors) in which no two processes in a group overlap their file views. Then, the con-

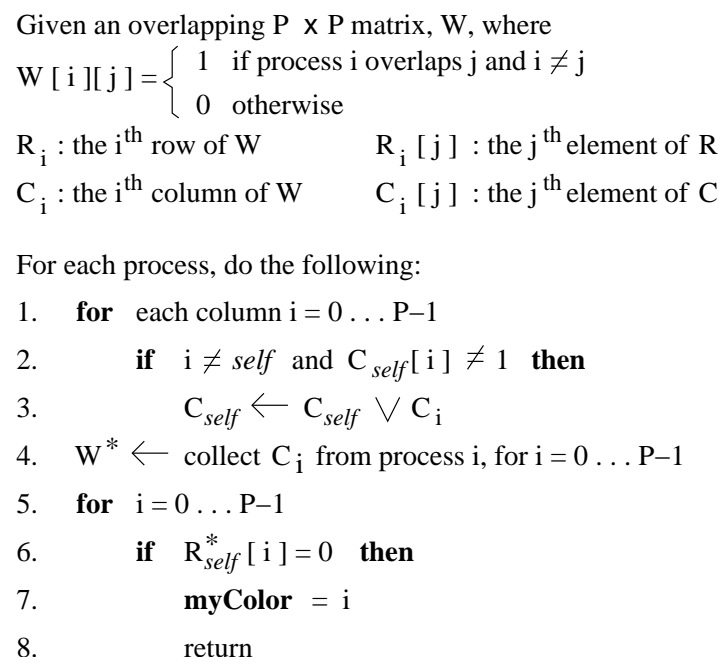

Figure 5: A greedy graph-coloring algorithm that finds the color id for each I/O process in variable myColor.

current $\mathrm{I} / \mathrm{O}$ is carried out in $k$ steps. Note that process synchronization between any two steps is necessary to ensure that no process in one group can proceed with its I/O before the previous group's I/O completes. The graph-coloring approach fulfills the requirement of MPI atomicity while maintaining at least a degree of I/O parallelism.

The graph-coloring methodology is a heuristic which has been studied for a long time and is proved to be NP-hard for general graphs [11]. Because the overlapping $\mathrm{I} / \mathrm{O}$ patterns present in most of the science applications are hardly arbitrary, a greedy solution may suffice. Figure 5 gives a simple greedy graph-coloring algorithm that first uses a $P \times P$ boolean matrix, $W$, to indicate if there is an overlap between two processes and starts coloring the processes by look- 

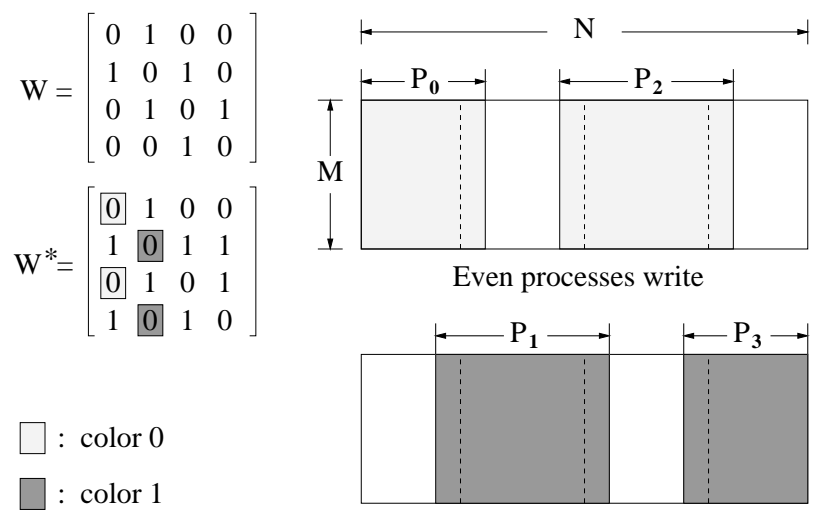

Odd processes write

Figure 6: An example of column-wise overlapping I/O using the graph-coloring approach. All I/O requests are performed in 2 steps: evenranked processes write at the first step followed by the writes from odd-ranked processes.

ing for the lowest ranked processes whose file views do not overlap with any process in that color. Let's now consider the column-wise partitioning example. Figure 6 shows the overlapping matrix using this greedy algorithm. It is obvious that for the two-dimensional columnwise partitioning case, two colors are enough to maintain MPI atomicity: the even-ranked processes perform their $\mathrm{I} / \mathrm{O}$ requests prior to the odd-ranked processes, as illustrated in Figure 6.

\subsubsection{Process-rank Ordering}

Another process-handshaking approach is to have all processes agree on a certain access priority to the overlapped file regions. An example is to use a policy where the higher ranked process wins the right to access the overlapped re-

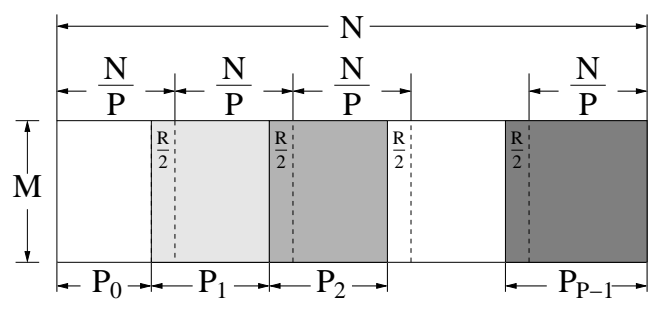

Process file views

Figure 7: The process file views for the columnwise overlapping I/O using process rank ordering approach. With the overlapping access removed, all $P \mathrm{I} / \mathrm{O}$ requests can be performed simultaneously while MPI atomicity is guaranteed.

gions while others surrender their writes. A couple of immediate advantages of this approach are the elimination of overlapping access and the reduction of the overall I/O amount. The overhead of this method is the re-calculation o each process's file view by marking down the overlapped regions with all higher-rank processes' file views.

Considering the column-wise partitioning example, Figure 7 illustrates the processes' file views when using the process-rank ordering approach. The file view for process $i, 0<i<$ $P-1$, is a $M \times \frac{N}{P}$ sub-array while the file views for processes 0 and $P-1$ are $M \times\left(\frac{N}{P}-\frac{R}{2}\right)$ and $M \times\left(\frac{N}{P}+\frac{R}{2}\right)$, respectively. Compared to Figure 6 , each process surrenders its write for the right-most $R$ columns. 


\subsection{Scalability Analysis}

In the column-wise partition case, the file locking approach results in $M N-\left(N-N^{\prime}\right)$ bytes, nearly the entire file, being locked while each process is writing. In fact, once a process is granted its write locking request, no other processes can access to the file. As a result, using byte-range file locking serializes the I/O and dramatically degrades the performance.

The purpose of proposing the two processhandshaking approaches is trying to maintain the I/O scalability without the use of file locking. The overhead of the graph-coloring approach is the communication cost of exchanging the file views, but this approach does not sacrifice I/O parallelism. The file views are used to construct the overlapping matrix locally. With a relative small negotiation overhead, the graphcoloring approach maintains a certain degree of I/O parallelism. To construct the overlapping matrix, the graph-coloring approach uses a logical bit to indicate if an overlap exists between two processes. In the process-rank ordering approach, the exact overlapped byte ranges must be know in order to calculate the local file view. This overhead is expected to be negligible when compared to the performance improvement resulting from the removal of all overlapping requests. Additionally, the overall I/O amount on the file system is reduced since the lower-rank processes surrender their accesses to the over-
Table 1: System configurations for the three parallel machines on which the experimental results were obtained.

\begin{tabular}{||c|c|c|c||}
\hline & Cplant & Origin 2000 & IBM SP \\
\hline \hline File system & ENFS & XFS & GPFS \\
\hline CPU type & Alpha & R10000 & Power3 \\
\hline CPU Speed & $500 \mathrm{MHz}$ & $195 \mathrm{MHz}$ & $375 \mathrm{MHz}$ \\
\hline Network & Myrinet & $\begin{array}{c}\text { Gigabit } \\
\text { Ethernet }\end{array}$ & $\begin{array}{c}\text { Colony } \\
\text { switch }\end{array}$ \\
\hline I/O servers & 12 & - & 12 \\
\hline $\begin{array}{c}\text { Peak I/O } \\
\text { bandwidth }\end{array}$ & $50 \mathrm{MB} / \mathrm{s}$ & $4 \mathrm{~GB} / \mathrm{s}$ & $1.5 \mathrm{~GB} / \mathrm{s}$ \\
\hline
\end{tabular}

lapped regions.

\section{Experiment Results}

We implemented the column-wise partitioning example using standard Unix I/O calls and obtained experimental results from three parallel machines: ASCI Cplant, an Alpha Linux cluster at Sandia National Laboratory; the SGI Origin 2000 at the National Center for Supercomputing Applications (NCSA); and Blue Horizon, the IBM SP at San Diego Supercomputing Center (SDSC). The machine configurations are briefly described in Table 1. Cplant is a Linux cluster running the Extended Network File System (ENFS) in which each compute node is mapped to one of the I/O servers in a round-robin selection scheme at boot time [12]. Basically, ENFS is an NFS file system with a few changes. The 

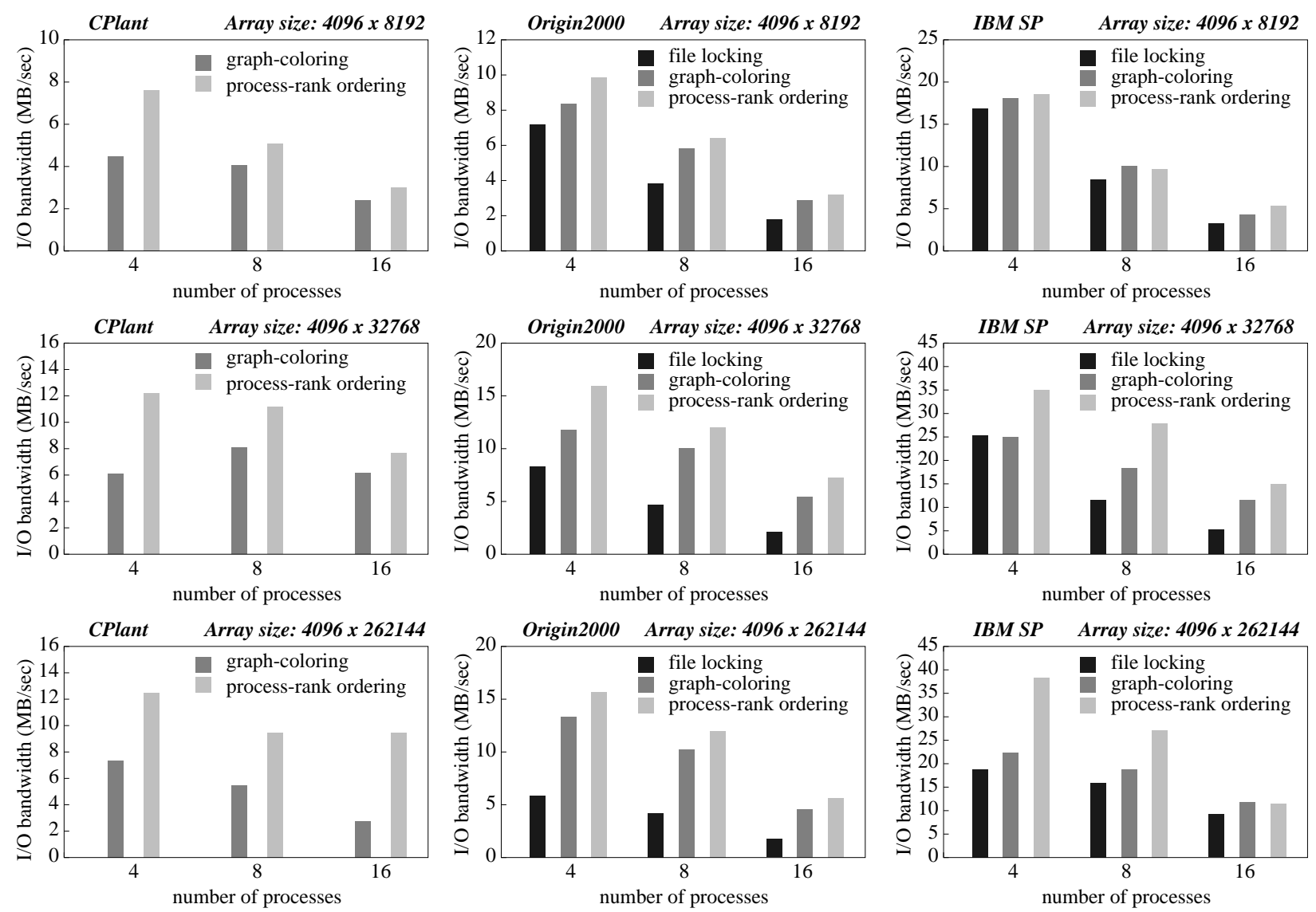

Figure 8: Performance results of running the column-wise partitioning experiments on a Linux Cluster, an IBM SP, and an SGI Origin200. Three file sizes were used: $32 \mathrm{MB}, 128 \mathrm{MB}$, and 1GB.

most notable is the absence of file locking on Cplant. Accordingly, our performance results on CPlant do not include the experiments that use file locking. ENFS also performs the optimization that NFS usually does, including readahead and write-behind.

We ran the experiments with the three array sizes: $4096 \times 8192(32 \mathrm{MB}), 4096 \times 32768(128$ $\mathrm{MB})$, and $4096 \times 262144(1 \mathrm{~GB})$. On all three machines, we used 4,8 , and 16 processors and the results are shown in Figure 8. Note the performance of file locking is the worst of the im- plementations of MPI atomicity. The poor results are also expected as discussed in Section 3.2 that file locking hinders the I/O concurrency. In most of the cases, the process-rank ordering strategy out-performed graph-coloring.

\section{Conclusions}

In this paper, we examined the atomicity semantics for both the POSIX and MPI specifications. The difference between them the number of non-contiguous regions in each $\mathrm{I} / \mathrm{O}$ requests. 
While POSIX considers only one contiguous file space I/O, a single MPI I/O request can access non-contiguous file space using MPI's file view facility. We compared a few implementation strategies for enforcing atomic writes in MPI including file locking, graph-coloring, and process-rank ordering. The experimental results showed that using file locking performed the worst when running a two-dimensional columnwise partitioning case. Since file locking is basically a central managed mechanism, the parallelism of concurrent $\mathrm{I} / \mathrm{O}$ requests, especially for overlapping I/O, can be significantly degraded by using it. The two alternatives proposed in this paper negotiate processes I/O request order of access priority through process handshaking. Without using a centralized locking mechanism, these two approach greatly improve the I/O performance.

The strategies of graph-coloring and processrank ordering require every process aware of all the processes participated in a concurrent I/O operation. In the scope of MPI, only collective calls have this property. Note that MPI collective $\mathrm{I} / \mathrm{O}$ is different from the concurrent $\mathrm{I} / \mathrm{O}$ in which a concurrent $\mathrm{I} / \mathrm{O}$ is for more general $\mathrm{I} / \mathrm{O}$ cases. An MPI non-collective I/O operation can also be concurrent. File locking seems to be the only way to ensure atomic results in noncollective I/O calls in MPI, since the concurrent processes are unknown. Otherwise, given the participating processes, I/O optimizations such as the process handshaking approach proposed in this paper can be applied to improve performance.

\section{Acknowledgments}

This work was supported in part by DOE laboratories, SNL, LANL and LLNL and in part by NSF EIA-0103023. It was also supported in part by NSF cooperative agreement ACI9619020 through computing resources provided by the National Partnership for Advanced Computational Infrastructure at the San Diego Supercomputer Center. We also acknowledge the use of the SGI Origin2000 at NCSA.

\section{References}

[1] P. Crandall, R. Aydt, A. Chien, and D. Reed. Input-Output Characteristics of Scalable Parallel Applications. In Supercomputing '95, Dec 1995.

[2] N. Nieuwejaar, D. Kotz, A. Purakayastha, C. Ellis, and M. Best. File-Access Characteristics of Parallel Scientific Workloads. IEEE Transactions on Parallel and Distributed Systems, 7(10):1075-1089, Oct 1996.

[3] E. Smirni, R. Aydt, A. Chien, and D. Reed. I/O Requirements of Scientific Applications: An Evolutionary View. In the 
Fifth IEEE International Symposium on High Performance Distributed Computing, pages 49-59, 1996.

[4] E. Smirni and D. Reed. Lessons from Characterizing the Input/Output Behavior of Parallel Scientific Applications. Performance Evaluation: An International Journal, 33(1):27-44, Jun 1998.

[5] IEEE/ANSI Std. 1003.1. Portable Operating System Interface (POSIX)-Part 1: System Application Program Interface (API) [C Language], 1996.

[6] IEEE Std. 1003.1-2001. System Interfaces, 2001.

[7] Message Passing Interface Forum. MPI-2: Extensions to the Message Passing Interface. http://www.mpiforum.org/docs/docs.html, July 1997.

[8] F. Schmuck and R. Haskin. GPFS: A Shared-Disk File System for Large Computing Clusters. In the Conference on File and Storage Technologies (FAST'02), pages 231-244, Jan 2002.

[9] Rajeev Thakur, William Gropp, and Ewing Lusk. Users Guide for ROMIO: A High-Performance, Portable MPI-IO Implementation. Mathematics and Computer Science Division, Argonne National Lab- oratory, October 1997. Technical Report ANL/MCS-TM-234.

[10] R. Thakur, W. Gropp, and E Lusk. On Implementing MPI-IO Portably and with High Performance. In the Sixth Workshop on I/O in Parallel and Distributed Systems, pages 23-32, May 1999.

[11] M. Garey and D. Johnson. Computers and Intractability: A Guide to the Theory of NP-Completeness. W.H. Freeman, New York, 1979.

[12] Sandia National Laboratory. Computational Plant. http://www.cs.sandia.gov/ Cplant. 